Journal for ImmunoTherapy of Cancer

\title{
DNA methylation-based prediction of response to immune checkpoint inhibition in metastatic melanoma
}

\author{
Katharina Filipski, ${ }^{1,2,3}$ Michael Scherer, ${ }^{4,5,6}$ Kim N. Zeiner, ${ }^{7}$ Andreas Bucher, ${ }^{8}$ \\ Johannes Kleemann, ${ }^{7}$ Philipp Jurmeister, ${ }^{9,10}$ Tabea I. Hartung, ${ }^{1}$ Markus Meissner, ${ }^{7}$ \\ Karl H. Plate, ${ }^{1,2,3}$ Tim R. Fenton (D) , ${ }^{11}$ Jörn Walter, ${ }^{4}$ Sascha Tierling, ${ }^{4}$ \\ Bastian Schilling (D) , ${ }^{12}$ Pia S. Zeiner (D) , 2,3,13 Patrick N. Harter ${ }^{1,2,3}$
}

To cite: Filipski K, Scherer M, Zeiner KN, et al. DNA methylation-based prediction of response to immune checkpoint inhibition in metastatic melanoma. Journal for ImmunoTherapy of Cancer 2021;9:e002226. doi:10.1136/ jitc-2020-002226

- Additional supplemental material is published online only. To view, please visit the journal online (http://dx.doi.org/10. 1136/jitc-2020-002226).

$\mathrm{KF}$, MS and KNZ are joint first authors.

PSZ and PNH are joint senior authors.

Accepted 25 June 2021

Check for updates

(C) Author(s) (or their employer(s)) 2021. Re-use permitted under CC BY-NC. No commercial re-use. See rights and permissions. Published by BMJ.

For numbered affiliations see end of article.

Correspondence to

Patrick N. Harter;

patrick.harter@kgu.de

\section{ABSTRACT}

Background Therapies based on targeting immune checkpoints have revolutionized the treatment of metastatic melanoma in recent years. Still, biomarkers predicting long-term therapy responses are lacking. Methods A novel approach of reference-free deconvolution of large-scale DNA methylation data enabled us to develop a machine learning classifier based on CpG sites, specific for latent methylation components (LMC), that allowed for patient allocation to prognostic clusters. DNA methylation data were processed using reference-free analyses (MeDeCom) and reference-based computational tumor deconvolution (MethylCIBERSORT, LUMP).

Results We provide evidence that DNA methylation signatures of tumor tissue from cutaneous metastases are predictive for therapy response to immune checkpoint inhibition in patients with stage IV metastatic melanoma. Conclusions These results demonstrate that LMC-based segregation of large-scale DNA methylation data is a promising tool for classifier development and treatment response estimation in cancer patients under targeted immunotherapy.

\section{BACKGROUND}

Malignant melanoma accounts for one of the most frequent cancer types and shows a rising incidence over the past years. ${ }^{1-3}$ Prognosis and treatment implications highly depend on the melanoma stage classified by the criteria of the American Joint Committee on Cancer (AJCC). ${ }^{45}$ The presence of distant metastases defines stage IV and thus advanced melanoma ${ }^{4}$ with patients facing a considerably poorer prognosis than in earlier melanoma stages. However, overall survival (OS) significantly improved due to novel therapy options. ${ }^{6}$ Among these, immune checkpoint inhibitors (ICI) modulating the anticancer immune response developed toward a promising treatment in tumors of various cancer entities. ${ }^{78}$ In metastatic melanoma, ICIs were approved as an effective therapy option. Of these, the combinatorial blockage of the immune checkpoints cytotoxic T-lymphocyteassociated protein 4 (ipilimumab) and programmed cell death protein 1 (PD-1, nivolumab/pembrolizumab) displayed the highest objective response rate in a phase III randomized clinical trial with $58 \%$ in the ipilimumab plus nivolumab arm compared with $19 \%$ in the ipilimumab monotherapy arm. ${ }^{9}$ Furthermore, multicenter studies have shown that anti-PD1-monotherapy (nivolumab, pembrolizumab) achieves response rates of up to $40 \%$ in treatment naïve patients and about $27 \%-35 \%$ in pretreated melanoma patients. $^{9-12}$ However, treatment resistance to ICIs remains a relevant issue. Equally important, serious immune-related adverse events (irAE) display a major challenge, particularly under combinatorial therapy which frequently lead to discontinuation of ICI treatment. ${ }^{13} 14$

Unfortunately, knowledge of biomarkers predicting response to ICI treatment ${ }^{15-17}$ or the occurrence of serious irAE is still limited. The expression of PD-1/PD-L1 (programmed death-ligand 1) as predictive biomarkers for treatment response to ICIs remains controversial $^{15} 16$ and lack of PD-L1 expression in melanoma is not necessarily associated with treatment failure. ${ }^{18}$ An innovative approach to investigate the relation between the immunological tumor microenvironment and ICI treatment response is the development of computational methods. These methods aim at processing and implementing largescale proteomic, metabolomic, transcriptomic, genetic and epigenetic data. As an example, the transcriptome-based algorithm Tumor Immune Dysfunction and Exclusion (TIDE) explores genes interfering with or promoting T-cell function. ${ }^{19}$ In this context, emerging genomic determinants of response to ICIs have been studied extensively. ${ }^{20}$ As a 
unique feature, epigenetic signatures are subjected to slower and sustained transformations within the dynamic changes of the tumor microenvironment. Certain DNA methylation patterns are cell-type-specific and therefore deconvolution of DNA methylation data enables the analysis of cellular composition ${ }^{21}$ as well as a precise brain tumor classification. ${ }^{22}$ With regard to potential epigenetic biomarkers, a particular DNA methylation profile among non-small cell lung cancer tissue samples from patients receiving ICI treatment was associated with an improved outcome, whereas mere PD-L1 expression, levels of tumor-infiltrating lymphocytes or the mutational load had no prognostic effects. ${ }^{23}$ Global differential DNA methylation patterns between melanoma samples might correlate with biological behavior and immunological processes. ${ }^{24}$ Moreover, immune cell methylation patterns might serve as prognostic biomarkers in melanoma. ${ }^{25}$ Taken together, there is still a lack of reliable biomarkers for the prediction of a successful therapy response after ICI treatment. DNA methylation profiling might be a robust approach for the development of prognostic or even predictive biomarkers in cancer, as so far, there is still a lack of reliable biomarkers for the prediction of a successful therapy response after ICI treatment.

In the current study, we investigated tumor DNA methylation patterns of two independent cohorts of melanoma patients using a novel computational approach of stepwise reference-free and reference-based tumor deconvolution with the aim to develop a machine learning classifier that allows for the prediction of a long-term therapy response in ICI-treated metastatic melanoma.

\section{METHODS}

\section{Sample selection and data acquisition of the ICI melanoma cohort}

The ICI cohort comprised cutaneous metastases of AJCC stage IV (eighth edition) melanoma patients treated in three different dermato-oncology centers (sites I-III) in Germany. Formalin-fixed and paraffin-embedded (FFPE) tissue samples (deriving from the biobank of the Department of Dermatology, Goethe-University, Frankfurt am Main, Germany, from the Institute of Pathology, Charité-Universitätsmedizin Berlin, Berlin, Germany and from the Department of Dermatology, University Hospital Würzburg, Würzburg, Germany) treated with ICIs between October 2010 and December 2020 were included. Clinical outcome was measured as survival under ICI therapy (defined as the time from start of ICI treatment to date of decease) and OS (defined as the time from primary diagnosis of melanoma to date of decease). Additionally, radiological response to ICI treatment was classified by Response Evaluation Criteria in Solid Tumours for immune-based therapies (iRECIST: iCPD, confirmed progressive disease; iUPD, unconfirmedPD; iSD, stable disease; iPR, partial response and iCR, complete response). Hereby, patients with disease control (DC, defined as iCR, iPR or iSD in at least one ICI treatment approach) were discriminated from patients with progressive disease (PD, defined as iUPD or iCPD). We additionally included the following parameters for each patient: epidemiological characteristics (sex, age), BRAF and NRAS mutation status and brain metastases status (yes vs no) (detailed information on the patient cohort and study workflow is depicted in figure 1 and online supplemental table 1). Data not passing well-established quality controls were excluded. In case of multiple samples per patient at site I, we selected the representative sample per patient according to the minimum euclidean distance of its methylation profile to the median profile (figure 1). Finally, the ICI cohort consisted of 65 samples from 65 patients (from sites I-III) of which 61 patients received complete radiological workup according to iRECIST (figure 1). The study protocol was endorsed by the local ethical committee (SNO-5-2019).

\section{Data acquisition of the TCGA melanoma cohort}

Clinical data of initially 470 patients suffering from melanoma were retrieved from The Cancer Genome Atlas (TCGA) data archive (TCGA SKCM). Clinical annotations included sex, age, AJCC tumor stages and site of tumor resection/biopsy. As the information on exact AJCC tumor stages (A, B, C and D) was missing in 98 cases, we annotated the simplified AJCC stages I-IV. Comprehensive data on ICI treatment or radiological therapy response were not eligible for this cohort. Corresponding raw intensity data (IDAT) files generated by use of the $450 \mathrm{k}$ Human Methylation Array (Illumina, San Diego, USA) were retrieved using the TCGA Genomic Data Commons tool. Data not passing well-established quality controls, patients with incomplete clinical annotations as well as melanoma in situ patients of AJCC stage 0 were excluded from the study resulting in 396 patients to set up the so-called TCGA melanoma cohort.

\section{Human methylation EPIC array of the ICI melanoma cohort}

While TCGA data were available as $450 \mathrm{k}$ Human Methylation array IDATs, for the ICI cohort of stage IV melanoma cutaneous metastases, we used the Human Methylation EPIC array (Illumina, San Diego, USA) comprising over 850000 CpG sites by analysis of representative tissue punches or macrodissected $10 \mu \mathrm{m}$ thick tumor slides from FFPE blocks. We followed standard protocols for tissue and DNA processing. Further processing of the microarrays and hybridization as well EPIC beadchip scanning were performed as indicated by the manufacturer.

\section{DNA methylation data processing}

DNA methylation data was obtained as IDAT files, which were used as input to the RnBeads software package. Quality control was performed using the built-in control probes on the EPIC array, and the data showed high overall quality. Furthermore, CpGs were filtered according to detection $\mathrm{p}$-values, and annotated single nucleotide polymorphisms, sites on the sex chromosomes and potentially cross-reactive sites were discarded from 
A

(1) Patient recruitment TCGA+Multicenter

(2) Sample acquisition $\mathrm{ICl}$ cohort

FFPE skin metastases

(3) Sample selection $\mathrm{ICl}$ cohort

(4) Patient stratification

(5) Analyses
$\mathrm{ICl}$ cohort

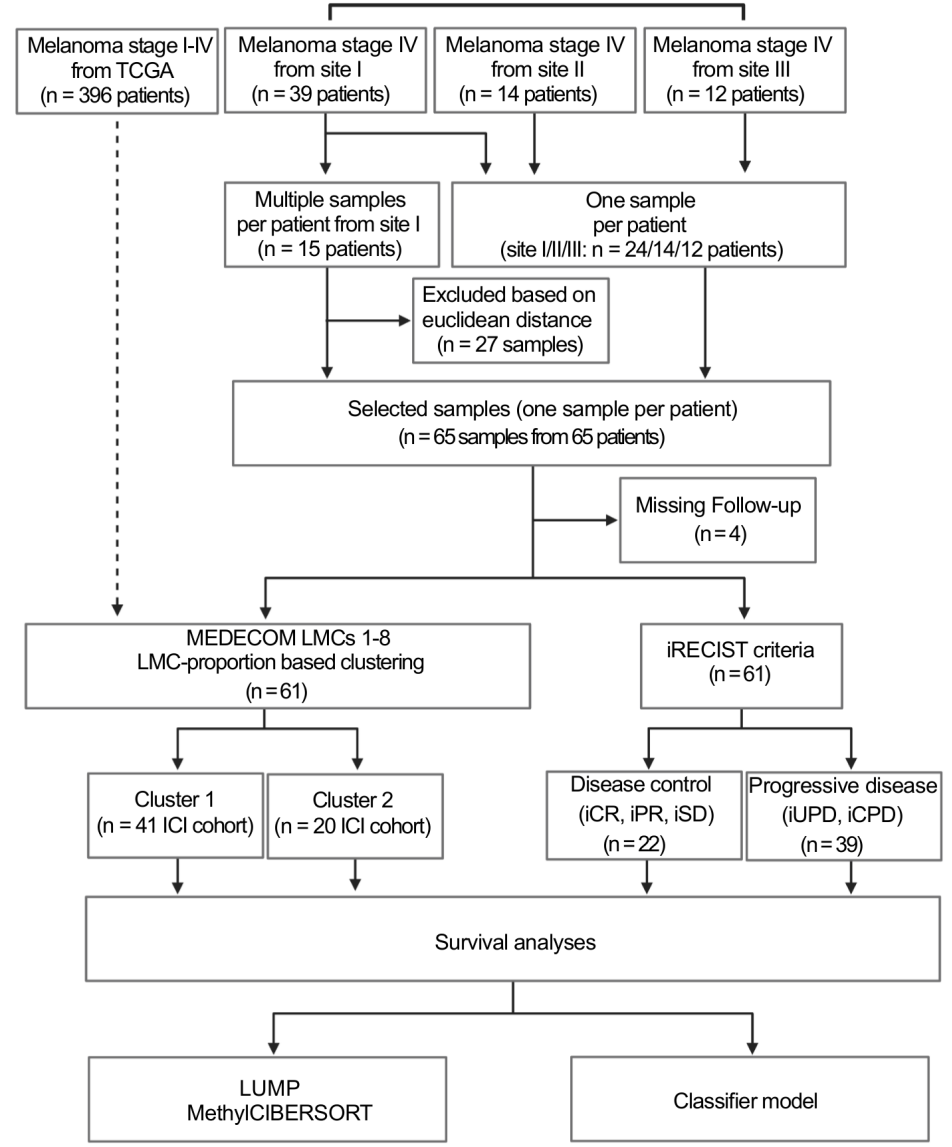

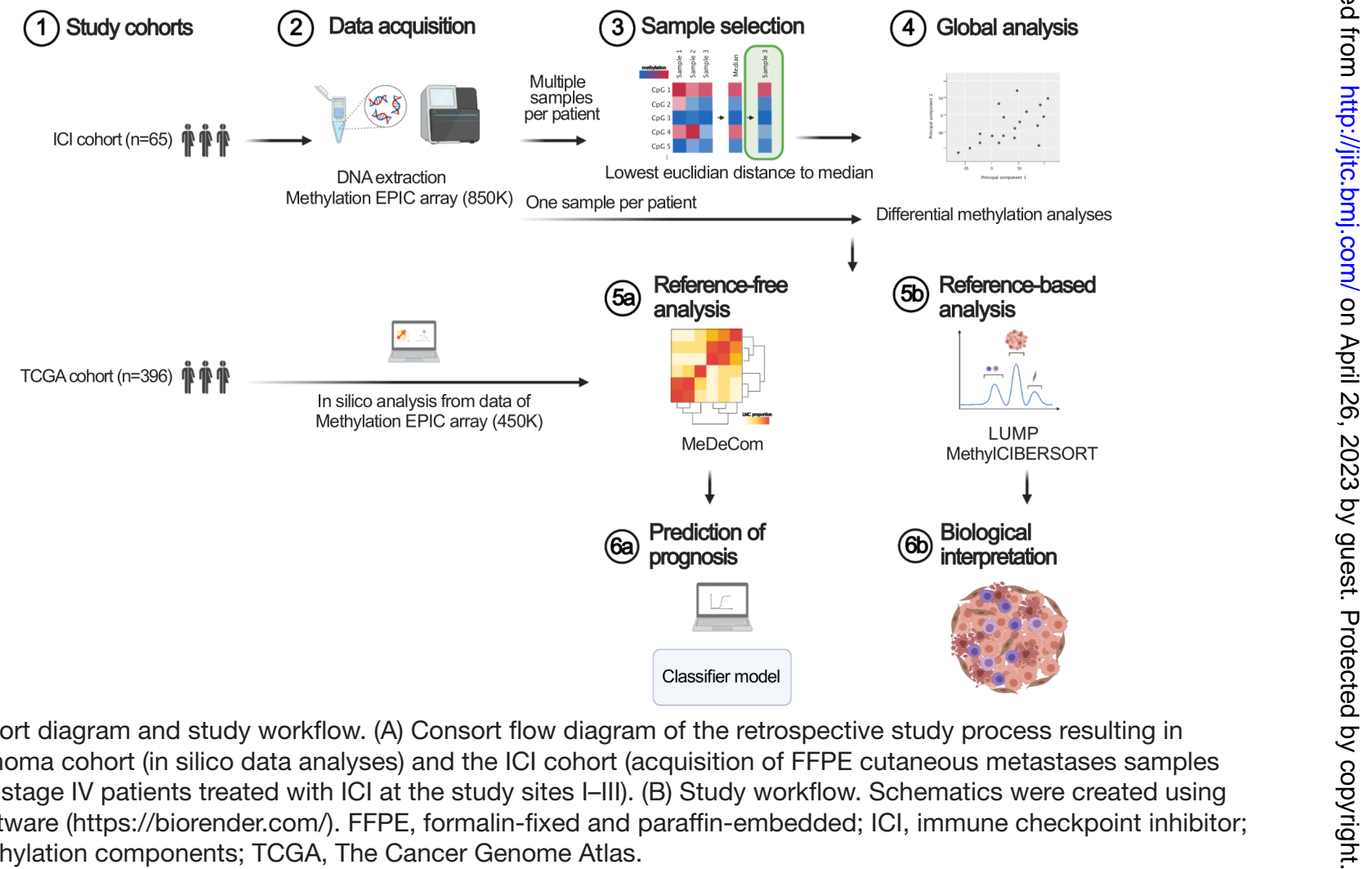


the analysis. ${ }^{26}$ Methylation data were normalized using the "dasen" method from the wateRmelon R-package. ${ }^{26}$ TCGA methylation data were processed analogously.

\section{Global DNA methylation analyses}

In a first approach, all eligible CpG sites passing quality controls in 65 tumor bulk samples were enrolled in principal component analysis (PCA). In addition, differential methylation analysis for all eligible CpG sites using RnBeads was performed comparing patient groups with different clinical characteristics like the melanoma subtype, BRAF or NRAS mutation status, absence or presence of brain metastases or radiological DC versus PD according to iRECIST. Raw data IDAT files as well as processed data of the ICI cohort are accessible via Gene Expression Omnibus (https://www.ncbi.nlm.nih.gov/ geo/; GSE175699).

\section{Reference-free MeDeCom analysis}

DNA methylation data of the bulk tumor samples of the TCGA melanoma cohort and the ICI cohort were investigated using the reference-free MeDeCom algorithm that dissects DNA methylation data into major components of variation, called latent methylation components (LMC). ${ }^{27}$ DNA methylation data of melanoma patients were processed according to a recently published protocol. ${ }^{28}$ The protocol selected the 5000 most variably methylated CpG sites across the samples as input to MeDeCom. Investigation of the cross-validation error and of the objective value for the parameter number of LMCs (kappa) and the regularization parameter (lambda) were performed, resulting in a set of LMCs. In order to prevent a strong dependence of the clustering on the LMC with the highest proportion across the samples, we decided to standardize the LMC proportions using z-scores. This allows for uncovering fine-grained changes in the LMC proportions and identification of subtle differences between the samples. LMC proportion values were standardized by subtracting the respective column mean and dividing by the column SD. Standardization was performed for LMCs 1-8 of all samples collectively (TCGA and ICI cohort), and separately in the TCGA and ICI cohort. Standardized LMC proportion-based clusters were further investigated regarding their prognostic and predictive significance. Hierarchical cluster analysis was performed using Ward's minimum variance method.

\section{Reference-based LUMP algorithm}

To estimate the leukocyte content in the bulk tumor samples, the leukocyte unmethylation for purity (LUMP) algorithm as implemented within RnBeads was employed. LUMP provides leukocyte ratios from the DNA methylation data by screening for $44 \mathrm{CpG}$ sites particularly hypomethylated in leukocytes. ${ }^{29} 30$

\section{Reference-based MethyICIBERSORT algorithm}

For a detailed deconvolution of the cellular composition of bulk melanoma samples, we applied the referencebased analysis MethylCIBERSORT. MethylCIBERSORT relies on DNA methylome-based reference data to infer distinct cellular contents (cancer cells, CD14-positive, CD19-positive, CD56-positive and CD8-positive cells, T regulatory cells, CD4-positive effector cells, eosinophils, fibroblasts and neutrophils). Methylation patterns of the sample of interest are compared with deposited celltype-specific and determinating DNA methylomes. ${ }^{31} 32$ MethylCIBERSORT analysis was carried out according to the respective protocols. ${ }^{32}$ Briefly, EPIC array IDAT sets were imported in R's "minfi" package to perform quality checks, Noob normalization and acquisition of beta values. Using the "MethylCIBERSORT" $\mathrm{R}$ package, a mixture file was built whose matrix consisted of beta values for comparison to a reference matrix (provided by TRF). This reference file contained signature methylation beta values of well-characterized cell types. After generating the mixture file, mixture and reference files were uploaded onto the CIBERSORT portal and deconvoluted (provided by the Alizadeh Lab, Stanford University, USA, developed by Newman $e t a l^{31}$ ).

\section{Patient classifier}

A patient classifier based on the 5000 most variable methylated CpGs selected for MeDeCom tumor deconvolution was developed for patient allocation to predictive LMC proportion-based clusters. Based on these CpG sites, we employed logistic regression using Lasso regularization (glmnet R-package) ${ }^{33}$ to predict whether a tumor sample would belong to the beneficial LMC proportionbased cluster or to the less beneficial cluster. Finally, this shrinkage led to 20 predictive CpGs. We employed nested 10 -fold cross-validation to estimate model performance and the hyperparameter lambda simultaneously.

\section{Statistics}

All statistical analyses were conducted using either JMP16 (SAS, Cary, USA) or R (R Core Team, 2019). The nonparametric Wilcoxon's test was applied for comparisons of data distributions between two groups. Kaplan-Meier survival curves were compared by log-rank and Wilcoxon's test. Likelihood ratios and risk ratios were computed in univariate proportional hazard models. Hierarchical clustering was performed by use of the Ward's minimum variance method.

\section{RESULTS}

\section{Workflow and cohort statistics}

To identify prognostic DNA methylation signatures in melanoma patients, we first performed global approaches for dimensional reduction of large-scale DNA methylome data. As a next step, we used reference-free and referencebased tumor deconvolution analyses, which allowed for development of a patient classifier and a biological interpretation of results (figure 1). The TCGA melanoma cohort comprised 396 tumor samples located in skin, soft tissue, central nervous system, peripheral, non-central nervous system organs and lymph nodes, respectively. 
A
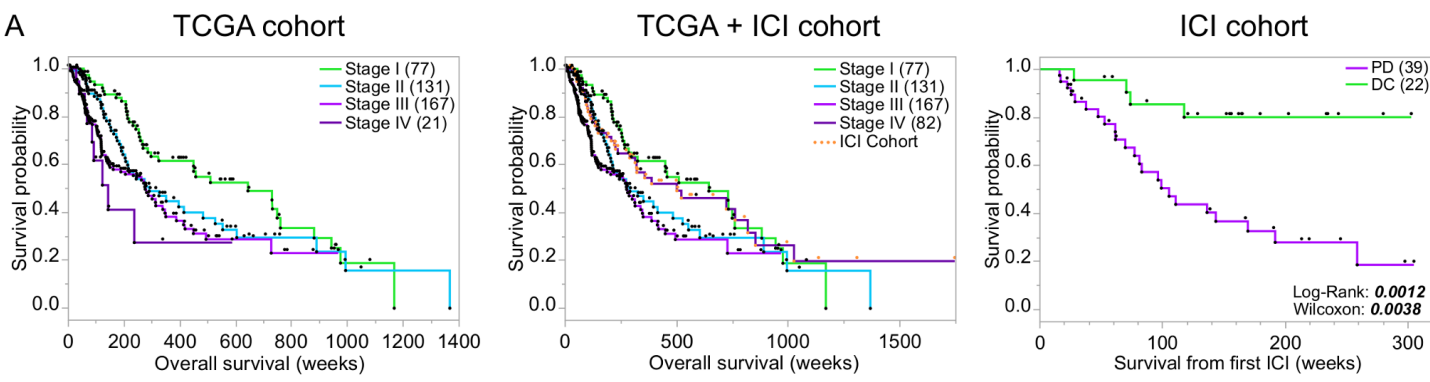

B TCGA $+\mathrm{ICl}$ cohort
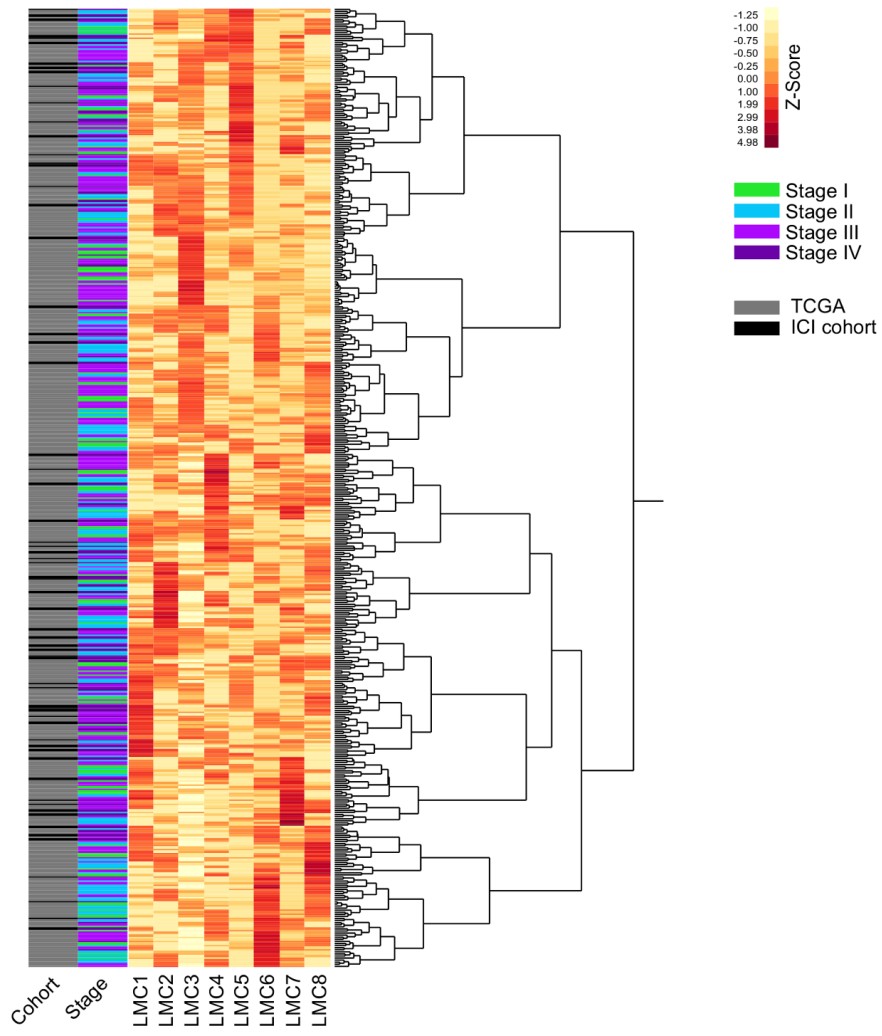

Figure 2 MeDeCom reference-free DNA methylome-based tumor deconvolution and standardized clustering of the total study population. (A) Kaplan-Meier survival curves indicating patient outcome characteristics of the different cohorts of the study: TCGA cohort (396 stages I-IV melanoma patients), total cohort (TCGA cohort plus ICl cohort including 65 patients with stage IV melanoma under ICI treatment (highlighted as orange dots)) and the ICl cohort discriminated by patients with progressive disease (PD) and disease control (DC) under ICI therapy, defined according to the neuroradiological iRECIST criteria. Survival times (weeks) were compared by log-rank and Wilcoxon test ( $p$-values depicted). (B) By use of the reference-free tumor deconvolution algorithm MeDeCom, eight LMCs were identified in the total patient cohort (TCGA+ICl cohort). Heatmap showing the standardized proportions of the LMCs in all patient samples of the total cohort (rows, $n=461$, cohort and melanoma stage depicted). Number of patients in parentheses. ICI, immune checkpoint inhibitor; LMC, latent methylation components; TCGA, The Cancer Genome Atlas.

Tumors from all AJCC tumor stages I-IV were included (figure 1). For validation of the TCGA cohort statistics, we performed Kaplan-Meier survival analysis which revealed that the applied simplified AJCC stages I-IV were prognostic (figure 2A). The ICI cohort included tissue from cutaneous metastases of 65 patients with AJCC stage IV melanoma treated with ICI therapy exclusively. Regarding the mutational status, $45.3 \%$ of the primary tumors showed a BRAF mutation, while $25.6 \%$ were $N R A S$ mutant. Brain metastases were observed in $40 \%$ of all patients (online supplemental table 1). We assessed OS, survival under ICI treatment as well as the radiological disease response assigning patients to either DC or PD. The Kaplan-Meier analysis of the survival under ICI therapy stresses a benefit in patients responding to ICI therapy according to the iRECIST criteria, thus proving cohort stringency (figure 2A).

\section{Global DNA methylation analysis does not decipher distinct signatures for melanoma subgroups}

In a first approach, we assessed global DNA methylation patterns including all eligible CpG sites that might differ between subgroups of the well-characterized cohort of stage IV melanoma patients treated with ICI. Using PCA 
neither $B R A F / N R A S$ mutational status, brain metastases status, nor radiological response status showed a clear association to the principal components (online supplemental figure 1A). Accordingly, differential methylation analysis did not reveal major differentially methylated CpGs (online supplemental figure 1B). This emphasizes that there is no global DNA methylation state associated with any of the available clinicopathological sample annotations.

\section{Reference-free MeDeCom analysis of the total study population and reference-based deconvolution algorithms of TCGA DNA methylation data}

Since the initial global DNA methylation analyses (PCA and differential methylation) of the stage IV melanoma cohort did not reveal an association with ICI therapy, we aimed for a more refined yet profound and reference-free algorithm to assess potential prognostic and predictive methylation signatures. Therefore, we first applied the MeDeCom algorithm to the bulk tumor DNA methylation data of the combined TCGA and ICI cohort (parameter selection is depicted in online supplemental figures 2). Overall, the cross-validation error pointed to selecting eight components (parameter $\mathrm{k}$ ) and the regularization parameter $\lambda$ as 0.001 . The proportions of the eight LMCs (LMC1-8) across the samples are visualized in figure 2B. Hierarchical cluster analysis of LMC proportion Z-scores of the entire cohort (TCGA+ICI) did not reveal clear clustering according to the cohort (TCGA vs ICI) and clusters did not show a clear association with AJCC tumor stages (figure 2B). In a next step, we analyzed the prognostic impact of LMC proportion-based clustering on OS of the TCGA cohort. Cluster analysis revealed two main clusters ( 1 vs 2 , figure $3 \mathrm{~A})$. Although, patients of cluster 1 showed a trend toward a better OS in the total TCGA cohort as well as in AJCG stages II and IV, log-rank and Wilcoxon's test did not indicate statistical significance (figure 3B). MeDeCom is a reference-free deconvolution algorithm of DNA methylation data, which generates LMCs and their proportions and follows dominant cell types. As we were interested in distinct cellular composition of LMC proportion-based clusters and as MeDeCom does not compare data to cell-type specific reference methylation profiles, we further investigated LMC proportion-based clusters 1 and 2 using the reference-based algorithms LUMP and MethylCIBERSORT.

Interestingly, the cancer cell fraction was significantly lower in the rather beneficial cluster 1 , whereas immune and stromal cells were strongly increased in cluster 1 (figure 3D and online supplemental figure 3A,B). Taking a closer look at immune cell subtypes, especially CD8positive T cells, B-cells, NK cells but also myeloid CD14positive cells were enriched in cluster 1. On the contrary, CD4-positive T cells were significantly reduced in cluster 1. In summary, this indicates that LMC proportion-based clusters might be of prognostic relevance in certain subgroups of melanomas in a heterogenous melanoma cohort. However, a precise interpretation of LMCs with regard to immune biological processes remains elusive.

Reference-free MeDeCom analysis of DNA methylation data exhibits predictive clusters in immune checkpoint inhibitor treated metastatic melanoma

In search of predictive signatures of large-scale DNA methylation data, we further investigated cutaneous metastases of stage IV melanoma patients receiving immune checkpoint inhibition in three dermato-oncology centers in Germany. Based on our initial MeDeCom analysis which decomposes DNA methylation data of the total cohort into eight LMCs, we performed LMC proportion-based unsupervised hierarchical cluster analysis in the ICI cohort only (figure 4A). Hierarchical cluster analysis depicted two major clusters with an enrichment of patients with DC in cluster 2 (figure 4A,B). In line with this, survival calculated from the initiation of ICI treatment was significantly longer in cluster 2 patients (figure 4C) and cluster 2 was predictive for survival from initiation of ICI treatment in univariate analysis (figure 4D). The median duration of ICI treatment as an indirect marker for DC and treatment tolerability accounted for 183 days in the beneficial cluster 2 as compared with 90 days in cluster 1 (Wilcoxon's non-parametric test $\mathrm{p}=0.03$ ).

Additional MethylCIBERSORT facilitated a microenvironmental analysis of the tumor samples allocating to the predictive LMC-based clusters in the ICI cohort. While the cancer cell fraction, as well as most immune cell subsets did not show significant differences between the beneficial cluster 2 and cluster 1, only regulatory $\mathrm{T}$ cells (Tregs), CD56-positive (NK) cells and fibroblasts were depleted in cluster 2 (figure $4 \mathrm{E}$ and online supplemental figure 3). Timing of biopsy (before vs under ongoing ICI treatment) did not have a substantial impact on the cellular composition of tumor bulks with solely the fraction of CD56-positive NK cells being enriched after initiation of ICI treatment (online supplemental figure 4). In summary, MeDeCom analysis unraveled predictive signatures for DC in metastatic melanoma being treated with ICI.

\section{LMC-based classifier allows for patient stratification into predictive clusters}

Finally, the findings of reference-free methylome analyses in the ICI cohort were integrated to develop a patient classifier with the aim to allocate patients to the predictive LMC proportion-based cluster. Therefore, we used the $5000 \mathrm{CpGs}$ computed by MeDeCom as an input for a logistic regression analysis. Lasso regularization revealed 20 of these $5000 \mathrm{CpG}$ sites to predict sample allocation to the less favorable cluster 1 or the favorable cluster 2 of the ICI cohort (figure 4F). By means of this classifier model, we achieved a cross-validated accuracy of $89.5 \%$ as well as an area under the curve of the receiver operating characteristic curve of 0.9664 in our cohort of melanoma stage IV patients under ICI treatment (figure 4G). 
A

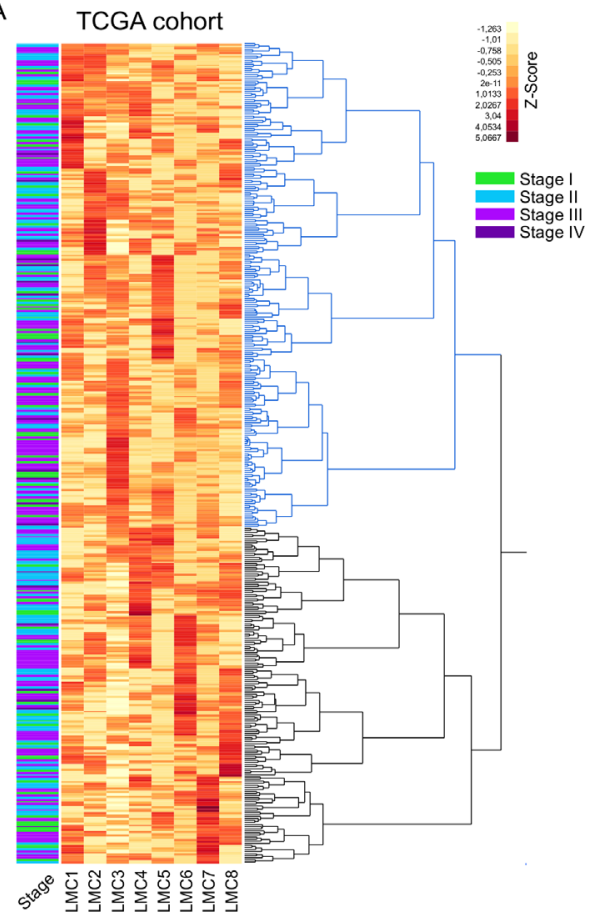

C
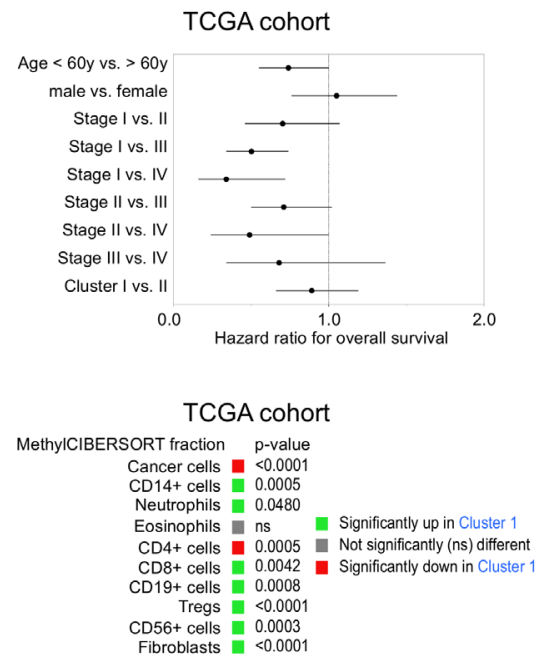
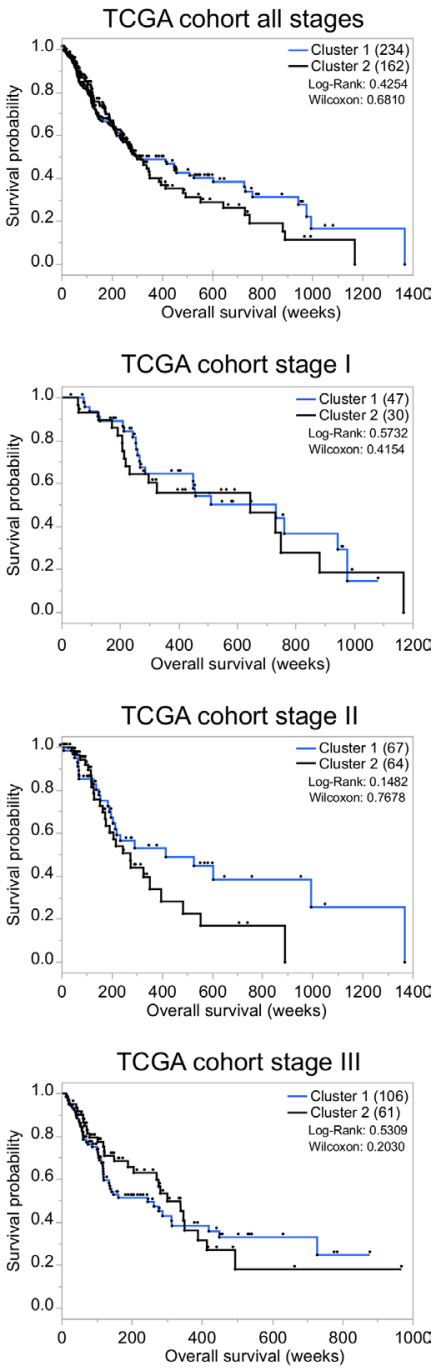

TCGA cohort stage IV

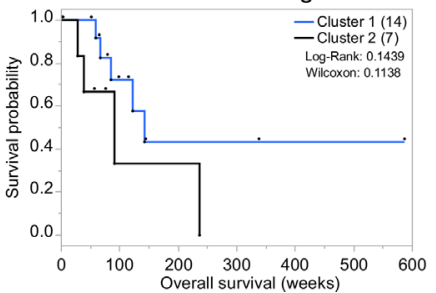

Figure 3 MeDeCom reference-free DNA methylome-based tumor deconvolution and standardized LMC-based clustering of the TCGA melanoma cohort. (A) Heatmap of the patient samples of the TCGA cohort (rows, $n=396$, melanoma stage depicted) showing the proportions of the eight LMCs that were previously identified by MeDeCom analysis of the total patient cohort (TCGA+ICl cohort) and then standardized in the TCGA cohort before clustering. Hierarchical clustering of standardized LMC values revealed two distinct clusters (1=blue, $2=$ black). (B) Kaplan-Meier survival curves regarding patient allocation to LMCbased cluster 1 vs 2 in the total TCGA cohort including all stages and in stages I-IV, respectively. Overall survival (weeks) was compared by log-rank and Wilcoxon test (p-values depicted). (C) Forest plot of TCGA cohort univariate proportional hazard analyses for the variables age at diagnosis, sex, melanoma stage and the LMC-based cluster 1 vs 2 . (D) Tumor deconvolution of the TCGA melanoma cohort was performed by the reference-based MethylCIBERSORT algorithm. The proportions of the respective cell fractions in melanoma samples of patients belonging to LMC-based cluster 1 (blue) were compared with patients belonging to LMC-based cluster 2 (black) by non-parametric Wilcoxon's test (significant p-values depicted). Number of patients in parentheses. ICl, immune checkpoint inhibitor; LMC, latent methylation components; TCGA, The Cancer Genome Atlas.

\section{DISCUSSION}

Malignant melanoma ranges among the top cancer diseases and its incidence is rising gradually. ${ }^{1-3}$ Immunomodulatory treatment approaches aiming to reinvigorate an exhausted lymphocytic immune response against tumor cells mainly by targeting immune checkpoints led to a substantial prognostic improvement in advanced melanoma. ${ }^{3435}$ Due to a limited response to ICI therapy 


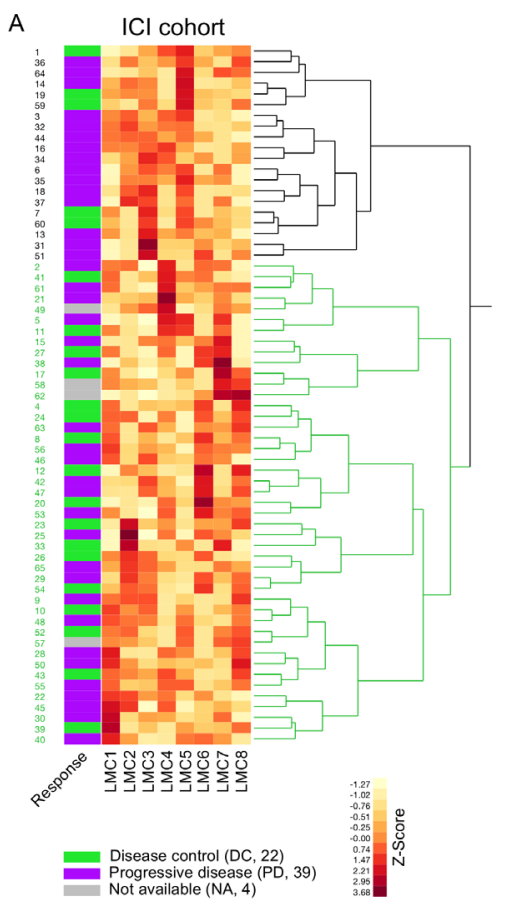

B

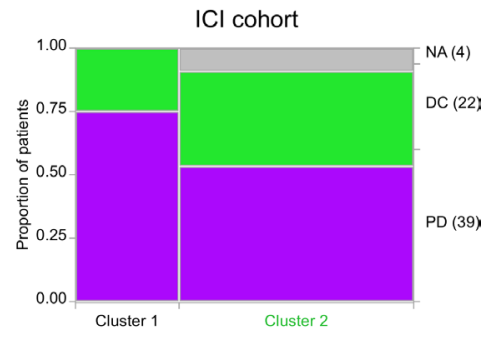

C

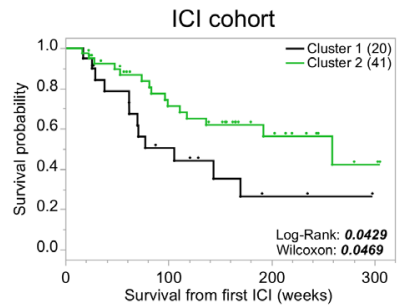

D

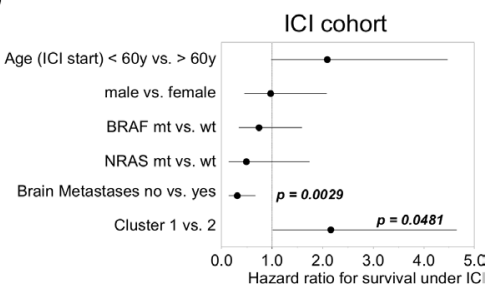

E $\mathrm{ICl}$ cohort MethyICIBERSORT fraction p-value Cancer cells in $\mathrm{ns}$ Neutrophils - ns Eosinophils = $\mathrm{ns}$ Significantly up in Cluster 2 CD4+ cells CD19+ cells ns Significantly down in Cluster 2 Tregs -0.0185
To $\begin{array}{ccc}\text { CD56+ cells } & 0.0283 \\ \text { Fibroblasts } & 0.0492\end{array}$

F

Classifier development 5000 CpGs in LMC 1-8

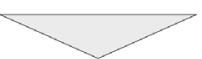

20 CpGs predictive for survival under $\mathrm{ICI}$

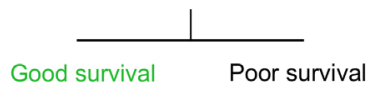

G

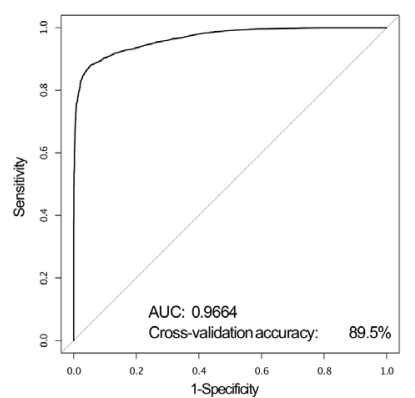

Figure 4 MeDeCom reference-free DNA methylome-based tumor deconvolution and standardized LMC-based clustering of stage IV melanoma patients under ICI therapy reveals predictive signatures. (A) Heatmap of the patient samples of the ICI cohort (rows, $n=65, I C l$ response defined by iRECIST depicted) showing the proportions of the eight LMCs that were previously identified by MeDeCom analysis of the total patient cohort (TCGA+ICl cohort) and then standardized in the ICl cohort before clustering. Hierarchical clustering of standardized LMC values revealed two distinct clusters (1=black, $2=$ green). (B) Proportion of patients with progressive disease (PD, purple) and disease control (DC, green) defined by iRECIST in cluster 1 and cluster 2 , respectively. Patients lost to iRECIST follow-up ( $n=4, N A=$ not available) were not included into further outcome analyses. (C) Kaplan-Meier survival curves separating patients allocated to LMC-based cluster 1 vs 2 of the ICl cohort. Survival from the start of ICl therapy (weeks) was compared by log-rank and Wilcoxon test ( $\mathrm{p}$-values depicted). (D) Forest plot of ICl cohort univariate proportional hazard analyses for the variables age at diagnosis, sex, BRAF and NRAS mutation status, brain metastasis status and the LMC-based cluster 1 vs 2 (significant $p$-values depicted). (E) Tumor deconvolution of the ICl cohort was performed by the reference-based MethylCIBERSORT algorithm. The proportions of the respective cell fractions in melanoma samples of patients belonging to the favorable LMC-based cluster 2 (green) were compared with patients belonging to LMC-based cluster 1 (black) by non-parametric Wilcoxon's test (significant p-values depicted). (F) Classifier development with (G) receiver operating characteristic curve of the prediction model. Number of patients in parentheses. AUC, area under the curve; ICl, immune checkpoint inhibitor; LMC, latent methylation components; TCGA, The Cancer Genome Atlas.

along with potentially severe irAEs, the identification of predictive biomarkers to improve patient selection for ICI treatment is crucial. Some microenvironmental and genomic predictors for ICI treatment response were identified over the past few years. ${ }^{20}$ These predictors include the expression of tumor antigens and neoantigens, the mutational burden, alterations in antigen presentation, DNA mismatch-repair and Interferon- $\gamma$ signaling, among others. ${ }^{20}$ Still, harmonization of applied assays, such as PD-L1 testing is pending. ${ }^{36}$ Predictive biomarkers cannot 
be reduced to single parts of the dynamic immune cascade and require the invention of more precise tools that are readily applicable in clinical routine. In this context, the analysis of epigenomic signatures especially DNA methylation-based approaches appear to be a promising candidate. ${ }^{2425}$

In the present study, we introduced a novel approach for the analysis of DNA methylation data from melanoma bulk samples by refining (i) global, (ii) reference-free and (iii) reference-based algorithms. This strategy turned out as a valid tool to decipher prognostic methylation signatures in melanoma patients and ultimately allowed the development of a classifier for ICI response in stage IV melanoma patients.

Global analyses of DNA methylation data failed to discriminate reliable prognostic or predictive signatures. This argued for a refinement of computational algorithms. By use of the reference-free tumor deconvolution algorithm MeDeCom, ${ }^{27}$ we were able to avoid the limitations imposed through a reference data $\operatorname{set}^{24}$ and gained a comprehensive view on the epigenetic tumor profiles. This approach is in contrast with previous studies that are mostly limited to reference-based tumor deconvolution algorithms which narrow down the amount of data entering the computation of prognostic methylation signatures. ${ }^{25}$ Based on clustering of LMC-proportion Z-scores, MeDeCom revealed signatures in stages I-IV melanoma patients of the TCGA cohort which were characterized by a distinct cellular composition but only showed a trend toward a prognostic relevance. The microenvironmental compositions of the samples were investigated by LUMP and MethylCIBERSORT representing reference-based deconvolution algorithms. ${ }^{21}{ }^{32}$ Chakravarthy et al were among the first to deconvolute tumors by means of DNA methylomes. ${ }^{32}$ Estimation of tumor purity and cellular microenvironmental composition allowed for allocation to immune hot and cold tumors. ${ }^{32}$ The association of the LMC-based clusters of the TCGA cohort with a particular microenvironmental composition might be relevant in earlier stage melanoma patients. However, the interpretation of outcome parameters in the TCGA cohort is limited due to the sampling heterogeneity including tissue from different organs, patients in different clinical stages (with only few stage IV patients) and only limited clinical annotations. Therefore, this aspect should be investigated in larger data sets of clinically harmonized melanoma cohorts. To investigate the predictive value of methylation signatures, we compiled a comparatively large cohort of stage IV melanoma patients that received ICI treatment. We were able to identify a predictive value of LMC proportion-based clusters correlating with survival under ICI treatment.

To understand potential biological mechanisms behind the predictive LMC proportion-based clusters, we amended reference-based deconvolution of the DNA methylation data.

In the ICI cohort, we did not find a distinct cell signature which was associated with the prognostic clusters and long-term DC. Recent studies reported the presence of B cells and so-called tertiary lymphoid structures to be associated with improved survival and a response to ICI therapy in melanoma. ${ }^{38}$ In contrast, Mitra et al investigated DNA methylation clusters based on median methylation values of selected immune cell-specific genes in melanoma patients but failed bringing forth an ICI response prediction signature..$^{25}$

The superior goal of a successful anticancer therapy is a long-term clinical benefit. Immunotherapy can lead to remarkable responses which can be objectified by imaging techniques. Unfortunately, even after showing an initial clinical or radiological response (assessed e.g. by the iRECIST), a considerable number of patients develop disease progression. Interestingly, our data show that LMC proportion-based clustering in ICI-treated melanoma can predict a durable long-term effect on ICI therapy reflected by a prolongation of survival after ICI treatment was initiated. Therefore, we aimed at integrating the findings to develop a pilot patient classifier that allowed to allocate melanoma patients under ICI treatment to the predictive LMC proportion-based clusters. This is a first step toward the development of a tool readily applicable in a clinical setup. At this early stage, it should be noted that our classifier has to be considered as a pilot tool established in a small patient cohort that yet has to be validated in larger, prospective settings to control for both clinical and radiological response parameters.

Taken together, our data demonstrate the benefit of reference-free deconvolution of DNA methylation data for cancer stratification. The obtained prognostic LMC proportion-based clusters have a very good predictive value for ICI treatment response in metastatic melanoma. The combined deconvolution approaches of bulk tumor DNA methylation data show a new way to develop a classifier to predict response to ICI treatment in melanoma. Further studies will be needed to validate this in larger cohorts of cancer patients treated with immunotherapy.

\section{Author affiliations}

${ }^{1}$ Neurological Institute (Edinger Institute), University Hospital, Frankfurt am Main, Germany

${ }^{2}$ German Cancer Consortium (DKTK) Heidelberg, German Cancer Research Center (DKFZ), Heidelberg, Germany

${ }^{3}$ Frankfurt Cancer Institute (FCl), Frankfurt am Main, Germany

${ }^{4}$ Department of Genetics, University of Saarland, Saarbrücken, Germany

${ }^{5}$ Max Planck Institute for Informatics, Saarland Informatics Campus, Saarbrücken, Germany

${ }^{6}$ Graduate School of Computer Science, Saarland Informatics Campus, Saabrücken, Germany

${ }^{7}$ Department of Dermatology, University Hospital, Frankfurt am Main, Germany ${ }^{8}$ Department of Radiology, University Hospital, Frankfurt am Main, Germany ${ }^{9}$ Institute of Pathology, Charité - Universitätsmedizin Berlin, corporate member of Freie Universität Berlin, Humboldt-Universität zu Berlin and Berlin Institute of Health, Berlin, Germany

${ }^{10}$ Institute of Pathology, Ludwig Maximilians University Hospital Munich, Munich, Germany

${ }^{11}$ School of Biosciences, University of Kent, Kent, UK

${ }^{12}$ Department of Dermatology, University Hospital Würzburg, Würzburg, Germany

${ }^{13} \mathrm{Dr}$. Senckenberg Institute of Neurooncology, University Hospital, Frankfurt am Main, Germany 
Acknowledgements We thank C Siedel, H Kneitz, M Dunst and T Starzetz for technical support. The results shown here are in part based on data generated by the TCGA Research Network: https://www.cancer.gov//tcga.

Contributors Conception and design of the work: KF, KNZ, PSZ, PNH. Acquisition, analysis and interpretation of data: KF, MS, KNZ, AB, JK, PJ, TIH, MM, KHP, TRF, JW, ST, BS, PSZ, PNH. Drafting of the manuscript: KF, MS, KNZ, PSZ, PNH. Critical revision for important intellectual content: KF, MS, KNZ, AB, JK, PJ, TIH, MM, KHP, TRF, JW, ST, BS, PSZ, PNH. Provided material and clinical data: KNZ, AB, JK, PJ, MM, KHP, BS. All authors approved the final version of the manuscript and agreed to all aspects of the work in ensuring that questions related to the accuracy or integrity of any part of the work are appropriately investigated and resolved.

Funding KF has received intramural funding by the Frankfurt Research Funding (FFF) program "Nachwuchswissenschaftler" and the "Clinician Scientist Program" by the Mildred-Scheel Foundation. MS is supported by the BMBF project de.NBI-epi (031L0101D) and the EU H2020 project SYSCID (733100). PSZ has received intramural funding by the FFF program "Nachwuchswissenschaftler" and "Patenschaftsprogramm" as well as within the "Clinician Scientist Program" by the Mildred-Scheel Foundation. The Dr Senckenberg Institute of Neurooncology is supported by the Dr Senckenberg Foundation. For this study, PNH obtained grants from "FCl/LOEWE: Discovery and Development Program".

Competing interests None declared.

Patient consent for publication Not required.

Ethics approval The retrospective study was approved by the local ethical committee (SN0-5-2019).

Provenance and peer review Not commissioned; externally peer reviewed.

Data availability statement Data are available in a public, open access repository. All data relevant to the study are included in the article or uploaded as supplementary information. All data analyzed and/or generated within this study are included in the article and the supplementary data files. Raw data IDAT files as well as processed data of the ICl cohort are accessible via Gene Expression Omnibus (GE0, https://www.ncbi.nlm.nih.gov/geo/ ; GSE175699). Any other relevant data are available upon reasonable request.

Supplemental material This content has been supplied by the author(s). It has not been vetted by BMJ Publishing Group Limited (BMJ) and may not have been peer-reviewed. Any opinions or recommendations discussed are solely those of the author(s) and are not endorsed by BMJ. BMJ disclaims all liability and responsibility arising from any reliance placed on the content. Where the content includes any translated material, BMJ does not warrant the accuracy and reliability of the translations (including but not limited to local regulations, clinical guidelines, terminology, drug names and drug dosages), and is not responsible for any error and/or omissions arising from translation and adaptation or otherwise.

Open access This is an open access article distributed in accordance with the Creative Commons Attribution Non Commercial (CC BY-NC 4.0) license, which permits others to distribute, remix, adapt, build upon this work non-commercially, and license their derivative works on different terms, provided the original work is properly cited, appropriate credit is given, any changes made indicated, and the use is non-commercial. See http://creativecommons.org/licenses/by-nc/4.0/.

\section{ORCID iDs}

Tim R. Fenton http://orcid.org/0000-0002-4737-8233

Bastian Schilling http://orcid.org/0000-0001-8859-4103

Pia S. Zeiner http://orcid.org/0000-0001-6626-9211

\section{REFERENCES}

1 Glazer AM, Winkelmann RR, Farberg AS, et al. Analysis of trends in US melanoma incidence and mortality. JAMA Dermatol 2017;153:225-6.

2 Little EG, Eide MJ. Update on the current state of melanoma incidence. Dermatol Clin 2012;30:355-61.

3 Miller KD, Nogueira L, Mariotto AB, et al. Cancer treatment and survivorship statistics, 2019. CA Cancer J Clin 2019;69:363-85.

4 Amin MB, Edge S, Greene F. AJCC cancer staging manual. 8th edn. New York: Springer International Publishing, 2017.

5 Swetter SM, Tsao H, Bichakjian CK, et al. Guidelines of care for the management of primary cutaneous melanoma. J Am Acad Dermatol 2019;80:208-50.

6 Luke JJ, Flaherty KT, Ribas A, et al. Targeted agents and immunotherapies: optimizing outcomes in melanoma. Nat Rev Clin Oncol 2017; 14:463-82.
7 Wolchok JD, Kluger H, Callahan MK, et al. Nivolumab plus ipilimumab in advanced melanoma. N Engl J Med 2013;369:122-33.

8 Robert C, Schachter J, Long GV, et al. Pembrolizumab versus ipilimumab in advanced melanoma. N Engl J Med 2015;372:2521-32.

9 Larkin J, Chiarion-Sileni V, Gonzalez R, et al. Five-Year survival with combined nivolumab and ipilimumab in advanced melanoma. $N$ Engl J Med 2019;381:1535-46.

10 Hamid O, Robert C, Daud A, et al. Five-year survival outcomes for patients with advanced melanoma treated with pembrolizumab in KEYNOTE-001. Ann Oncol 2019;30:582-8.

11 Robert C, Ribas A, Schachter J, et al. Pembrolizumab versus ipilimumab in advanced melanoma (KEYNOTE-006): post-hoc 5-year results from an open-label, multicentre, randomised, controlled, phase 3 study. Lancet Oncol 2019;20:1239-51.

12 Larkin J, Minor D, D'Angelo S, et al. Overall survival in patients with advanced melanoma who received nivolumab versus investigator's choice chemotherapy in CheckMate 037: a randomized, controlled, open-label phase III trial. J Clin Oncol 2018;36:383-90.

13 Postow MA, Sidlow R, Hellmann MD. Immune-Related adverse events associated with immune checkpoint blockade. N Engl J Med 2018;378:158-68.

14 Hodi FS, Chiarion-Sileni V, Gonzalez R, et al. Nivolumab plus ipilimumab or nivolumab alone versus ipilimumab alone in advanced melanoma (CheckMate 067): 4-year outcomes of a multicentre, randomised, phase 3 trial. Lancet Oncol 2018;19:1480-92.

15 Liu D, Jenkins RW, Sullivan RJ. Mechanisms of resistance to immune checkpoint blockade. Am J Clin Dermatol 2019;20:41-54.

16 Sharma P, Allison JP. The future of immune checkpoint therapy. Science 2015;348:56-61.

17 Patel SP, Kurzrock R. PD-L1 expression as a predictive biomarker in cancer immunotherapy. Mol Cancer Ther 2015;14:847-56.

18 Grosso J, Horak CE, Inzunza D, et al. Association of tumor PD-L1 expression and immune biomarkers with clinical activity in patients (pts) with advanced solid tumors treated with nivolumab (anti-PD-1; BMS-936558; ONO-4538). J Clin Oncol 2013;31:3016.

19 Jiang P, Gu S, Pan D, et al. Signatures of T cell dysfunction and exclusion predict cancer immunotherapy response. Nat Med 2018;24:1550-8.

20 Keenan TE, Burke KP, Van Allen EM. Genomic correlates of response to immune checkpoint blockade. Nat Med 2019;25:389-402.

21 Houseman EA, Accomando WP, Koestler DC, et al. DNA methylation arrays as surrogate measures of cell mixture distribution. BMC Bioinformatics 2012;13:86.

22 Capper D, Jones DTW, Sill M, et al. DNA methylation-based classification of central nervous system tumours. Nature 2018;555:469-74.

23 Duruisseaux M, Martínez-Cardús A, Calleja-Cervantes ME, et al. Epigenetic prediction of response to anti-PD-1 treatment in nonsmall-cell lung cancer: a multicentre, retrospective analysis. Lancet Respir Med 2018;6:771-81.

24 Lauss $M$, Ringnér $M$, Karlsson $A$, et al. DNA methylation subgroups in melanoma are associated with proliferative and immunological processes. BMC Med Genomics 2015;8:73.

25 Mitra S, Lauss M, Cabrita R, et al. Analysis of DNA methylation patterns in the tumor immune microenvironment of metastatic melanoma. Mol Oncol 2020;14:933-50.

26 Pidsley R, Y Wong CC, Volta M, et al. A data-driven approach to preprocessing illumina $450 \mathrm{~K}$ methylation array data. BMC Genomics : 2013;14:293.

27 Lutsik P, Slawski M, Gasparoni G, et al. MeDeCom: discovery and quantification of latent components of heterogeneous methylomes. Genome Biol 2017;18:55.

28 Scherer M, Nazarov PV, Toth R, et al. Reference-free deconvolution, visualization and interpretation of complex DNA methylation data using DecompPipeline, MeDeCom and FactorViz. Nat Protoc 2020;15:3240-63.

29 Aran D, Sirota M, Butte AJ. Systematic pan-cancer analysis of tumour purity. Nat Commun 2015;6:8971

30 Müller F, Scherer M, Assenov Y, et al. RnBeads 2.0: comprehensive analysis of DNA methylation data. Genome Biol : 2019;20:55.

31 Newman AM, Liu CL, Green MR, et al. Robust enumeration of cell subsets from tissue expression profiles. Nat Methods 2015;12:453-7.

32 Chakravarthy A, Furness A, Joshi K, et al. Pan-cancer deconvolution of tumour composition using DNA methylation. Nat Commun 2018;9.

33 Friedman J, Hastie T, Tibshirani R. Regularization paths for generalized linear models via coordinate descent. J Stat Softw 2010;33:1-22.

34 Wolchok JD, Chiarion-Sileni V, Gonzalez R, et al. Overall survival with combined nivolumab and ipilimumab in advanced melanoma. $N$ Engl J Med 2017;377:1345-56. 
35 Schachter J, Ribas A, Long GV, et al. Pembrolizumab versus ipilimumab for advanced melanoma: final overall survival results of a multicentre, randomised, open-label phase 3 study (KEYNOTE-006). Lancet 2017:390:1853-62.

36 Hansen AR, Siu LL. PD-L1 testing in cancer: challenges in companion diagnostic development. JAMA Oncol 2016;2:15-6.
37 Cabrita R, Lauss M, Sanna A, et al. Tertiary lymphoid structures improve immunotherapy and survival in melanoma. Nature 2020;577:561-5.

38 Helmink BA, Reddy SM, Gao J, et al. B cells and tertiary lymphoid structures promote immunotherapy response. Nature 2020;577:549-55. 